OPEN ACCESS

Edited by:

Renato de Filippis,

University of Catanzaro, Italy

Reviewed by:

Hatice Eken,

Vanderbilt University, United States

*Correspondence:

Mireia Solerdelcoll

mireia.solerdelcoll_arimany@kcl.ac.uk orcid.org/0000-0003-2250-494X

Specialty section:

This article was submitted to

Public Mental Health,

a section of the journal

Frontiers in Psychiatry

Received: 22 December 2021

Accepted: 13 January 2022

Published: 10 February 2022

Citation:

Solerdelcoll M (2022) A Wake-Up Call:

Recognizing and Reimaging

Responses to Children's Mental

Health and Protection Needs as an

Integral Part of the COVID-19

Pandemic.

Front. Psychiatry 13:841515

doi: 10.3389/fpsyt.2022.841515

\section{A Wake-Up Call: Recognizing and Reimaging Responses to Children's Mental Health and Protection Needs as an Integral Part of the COVID-19 Pandemic}

\author{
Mireia Solerdelcoll ${ }^{1,2 *}$ \\ ${ }^{1}$ Department of Child and Adolescent Psychiatry, Institute of Psychiatry, Psychology and Neuroscience, King's College \\ London, London, United Kingdom, ${ }^{2}$ Department of Medicine, University of Barcelona, Barcelona, Spain
}

Keywords: COVID-19, child development, child protection, mental health, culture, resilience, vulnerable population

\section{INTRODUCTION}

The coronavirus disease 2019 (COVID-19) pandemic is an unprecedented worldwide crisis that presents unprecedented risks to the safety, wellbeing and development of the world's children. The pandemic-related economic breakdown and psychosocial impact have increased the existing child psychosocial needs and highlighted the weaknesses of mental health systems globally (1). Moreover, the harmful effects of COVID-19 and related control measures are not distributed equally, and the most damaging and longest-lasting consequences are likely to impact those who are already most disadvantaged or vulnerable (2). The pandemic is also exposing the fact that the harmful effects are not equally distributed and particular vulnerable and already disadvantaged groups, such as children from low-income and middle-income countries (LMICs), those with neuropsychiatric conditions or disabilities, and those in vulnerable social circumstances (for instance, refugee or migrant children, and children living in alternative care or overcrowded settings), might be at even greater risk of poor educational, social and mental health outcomes because pre-existing failures in human rights protection are worsened $(3,4)$.

If the pandemic has taught us anything, it is that mental health is profoundly affected by the world around us. Socio-economic and cultural factors and humanitarian crises like the COVID-19 pandemic can lead to severe and lasting psychosocial distress (5). Children are subject to multiple influences throughout their lives, and mental health is also a reflection of their circumstances, personal experiences, and cultural context (6). This opinion article provides an overview of the foundational role of culture and environmental contexts on children's mental health and wellbeing under the COVID-19 circumstance and discusses recommendations and considerations on how culturally sensitive approaches can promote and protect mental health and care for the most vulnerable youth in these challenging times.

\section{SPHERES OF INFLUENCE ON CHILDREN'S MENTAL HEALTH AND WELLBEING}

Culture and contexts shape how mental disorders are experienced, understood and addressed. For children and young people understanding mental health means recognizing that is entwined with societal and family values, cultural standards, social expectations, and developmental capacities (7). For example, perceptions of acceptable behavior, appropriate social functioning, expectations of 
happiness and personal growth, and experiences of adversity are understood differently in different cultural contexts, affecting understanding of mental health and wellbeing. As a result, the successful implementation of mental health policies and programmes need to take into consideration familiar, cultural, social, political, and environmental contexts. When these different perspectives and cultural contexts inform responses to mental health, they can lead to prioritizing interventions that are more beneficial, acceptable and that build on the strengths of those societies (8).

Over time and as the pandemic unfolds, children are subject to multiple influences that lead to positive and negative trajectories. These differences often stem from cultural stereotypes and norms, powerful social determinants of mental health, but are also linked to family and community behaviors and expectations (9).

Recognizing a socio-ecological framework, multiple factors shape the mental health of children and youth (10). It is molded by the worlds of parents and caregivers, communities and schools, and large-scale social determinants such as poverty, conflict, and pandemics (11). At critical moments of child development, these experiences and environments can contribute to pre-existing vulnerabilities and harm mental health and wellbeing, but they also offer unique opportunities to promote and protect mental health and resilience $(7,12)$. A recent UNICEF report synthesized six life domains that have the greatest impact on children's development: physical and mental health and wellbeing; economy and equality; learning and human capital formation; violence and conflict; family relationships and social networks (9).

\section{THE ROLE OF PARENTING IN ADDRESSING MENTAL HEALTH AND PSYCHOSOCIAL ASPECTS}

The role of parenting is foundational to children's mental health (13). Positive parenting skills become even more important when schools are closed, social services are disrupted, and children are confined at home. Positive parenting can foster adolescents' capacity for resilience in the face of adversity and have longlasting effects on health and education. Across diverse cultural contexts, warm relationships between caregivers and children can lead to positive outcomes, including higher self-esteem, reduced stress, better mental health and fewer psychological and behavior problems $(14,15)$. However, parents' psychological distress reduces their parenting skills and leads to impaired parent-child interactions (16). A key approach to laying the foundation for youth mental health requires providing care for caregivers and encouraging engaged parenting. To achieve good mental wellbeing as part of COVID-19 response efforts, we must ensure that the family's basic needs are met and that the rights of children are fully promoted and protected. Supporting parents and caregivers should be addressed through collaborative

Abbreviations: COVID-19, coronavirus disease 2019; LMICs, low-income and middle-income countries. networks between key actors such as governments and local authorities, intergovernmental organizations, community-based and civil society actors, and healthcare professionals, by the implementation of social protection measures that include financial and nutritional support, family-friendly policies, and parent training and counseling programs. When social inequalities remain unaddressed, mental health interventions are less effective. Thus, inequality is an important determinant of mental health, and it is worsening as a result of the pandemic. Mental health and psychosocial response require recognition of the syndemic co-occurrence and interaction of health burdens and sociocultural context (17). Family environments marked by poverty or limited resources will bear the full brunt of COVID19 and associated containment measures (3). High-stress home environments increase the likelihood of family conflicts, as well as emotional and behavioral problems, domestic abuse and violence. Therefore, the effectiveness of public health strategies and programmes in global mental health should strategically address the social determinants (6). Enabling families to cope with this situation will require reducing stressors and economic instability and increasing parenting capabilities, along with mental health support. An effective COVID-19 response promotes responsive caregiving and nurturing connections, ensures mental health care for caregivers, and provides parents training to respond to children's mental health challenges. Mental health programmes must prioritize caregivers by providing support to manage stress and conflict and enhancing coping strategies, prioritizing targeted support for families and children at particular risks, such as those facing violence and groups with previous vulnerabilities (e.g., mental and substance use disorders) (18).

\section{EDUCATION ENVIRONMENTS}

Information, education, and online materials on mental health and psychosocial wellbeing have been produced by many institutions and organizations to provide immediate help and coping strategies for managing infection containment-related psychological stress during the COVID-19 pandemic and beyond (19). Despite the window of opportunity presented by the use of digital technology for various mental health care tasks, caution is needed as barriers to access and use of different telehealth resources may increase existing disparities in access to mental health care. Two-thirds of the world's school-age children have no internet connection at home and in the context of COVID19 and prolonged school closures, it can lead to isolation, loss of learning, and perpetuate inequalities that already divide youth from the poorest households, rural areas, and LMICs from their peers. Aside from the important academic benefits of schooling, schools have an essential role in shaping the mental wellbeing of young people by providing a structured and supervised space for socioemotional development, friendship and social support networks, protection from risk-taking behaviors, and often represent a key access point for food, which negatively affects mental health (1). 
Schools provide a critical platform to promote and protect children's and young people's mental health and reach children in need of care, those who might otherwise not have access to mental health services. In countries at all income levels, especially in LMICs, evidence shows that school-based interventions that focus on developing social, emotional, problem-solving and coping skills are linked to mental health benefits. As a result, learning environments are critical platforms where cost-effective and culturally acceptable interventions can foster inclusion and promote and protect mental health $(20,21)$.

We suggest that an inter-agency collaboration between child protection actors, education, and mental health professionals should be adopted in the design and development of programs for preventing, identifying, and delivering psychosocial support to children and adolescents and their caregivers (22).

\section{EXPERIENCES OF STIGMA AND DISCRIMINATION}

Despite growing media and community awareness of the importance of mental health and the establishment of children's mental health as a core priority of governments and other stakeholders, it is still widely stigmatized and misunderstood. The influences of stigma on mental health are complex and can work bi-directionally, instigating and exacerbating mental health conditions (23). Additionally, stigma can intersect and combine with other forms of discrimination, such as gender, race, socioeconomic status, sexual orientation, or disability, to lead to poor mental health and hamper efforts to promote mental health and protect vulnerable children and youth. Mental health is widely stigmatized and influenced by laws, policies, the media, attitudes, and cultural norms that perpetuate stereotypes and limit opportunities for children and youth to grow, learn, and prosper $(24,25)$.

Against a backdrop of rising awareness of mental health issues, there is now an opportunity to promote good mental health for every child, protect vulnerable children, and care for children facing the greatest challenges. Making that happen will require not only investment in mental health across multiple sectors, including primary health care, education, and social protection sectors, but also requires societies to break the silence surrounding mental health, by addressing stigma, promoting understanding and a positive state of wellbeing for children and their caregivers (6).

Racial discrimination devalues, disempowers and, whether felt directly or indirectly, significantly harms children's mental health and wellbeing. Research suggests that the effect of racism on mental health is profound, complex, entrenched and pervasive $(26,27)$. Racism is a social determinant of health that has a profound impact on health status and affects mental health in multiple ways: by disparities in educational access and outcomes; increased and prolonged levels of exposure to stress; the internalization of negative stereotypes related to race and damaging self-esteem; disadvantage and discrimination as a factor limiting the access to health care, and can also impact the diagnosis of mental health conditions. Perceived racial discrimination is associated with increased symptoms of depression, anxiety, post-traumatic stress disorder, poor sleep, reduced self-regulation, and increased rates of risky behaviors, substance use or delinquency. Additionally, experiences of racism can result in a downstream effect of racial inequality, such as intergenerational and historical trauma (27). Thus, recognizing the cultural background as an integral part of the person and addressing the roots of discrimination is essential to safeguard mental health. A core commitment to a culturally sensitive approach to mental health care for young people and adjusted to their particular needs and challenges must be a guiding principle. Recent mental health guidelines call on practitioners to raise their awareness of discrimination; conduct culturally aware assessments; establish an individualized and culturally appropriate treatment approach; and provide multidisciplinary assistance (28).

\section{RESILIENCE}

Human resilience depends on a combination of multiple biological, psychological, social, and ecological systems interacting as a combination of co-occurring and co-dependent elements in ways that help individuals to regain, sustain, or improve their mental wellbeing in contexts of adversity (29). In the context of exposure to adversity, Ungar argued that resilience is both the capacity of individuals to navigate their way to the psychological, social, cultural, and physical resources that sustain their wellbeing, and their capacity individually and collectively to negotiate for these resources to be provided and experienced in culturally meaningful ways (30). Resilience requires the ability to navigate seven tensions: access to material resources; relationships; power and control; social justice and cohesion; and being especially relevant for this opinion article: a personal and collective sense of identity, purpose and belonging; and cultural adherence and cohesion to local, global or cultural practices, beliefs, including religious beliefs, and values (31). Fostering resilience in children and youth requires recognizing the unique qualities in diverse cultural forms with specific values and concepts of mental health, needs, and resources (32).

While the measures adopted by public health authorities to prevent and control the spread of COVID-19 were necessary to reduce the disease burden, accumulating evidence suggests that pandemic-related restrictions have resulted in an increase in known risk factors for mental health problems and have contributed to the risk of deepening social, educational, and health inequalities, exacerbating pre-existing vulnerabilities for marginalized and disadvantaged children and families (33). Overall, the data published to date suggest that while some children demonstrated resilience, a significant cohort of children and adolescents appear to be experiencing psychological distress related to the COVID-19 crisis $(34,35)$. As the pandemic has progressed, referrals to child mental health services have increased, reaching record demands (36).

Studies of resilience show that regulatory capacities and changes to cognitions are unsustainable unless other cooccurring social and physical resources, such as family, 
housing, and natural environment are adequate and facilitated (37). The promotive and protective factors and processes enable resilience when they express sensitivity to contextual and cultural dynamics. Thus, heightened awareness of how resilience interventions can meaningfully respond to the COVID-19 pandemic has the potential to enable mental health practitioners to better support positive mental health outcomes in children. To this end, mental health professionals need to work in multidisciplinary teams to promote interventions that promote resilience tailored to the cultural and contextual norms of different populations who can facilitate access to protective socioecological supports while treating disorders (38).

\section{CONCLUSIONS AND FUTURE DIRECTIONS}

To promote good mental health for every child, protect vulnerable children and care for children facing the greatest challenges, we outline a framework on the foundation of three core principles: commitment to strengthening leadership and partnerships, backed by investment in supporting mental health; Communication to break down stigmas around mental health, opening conversations and improving mental health literacy; and Action to strengthen the capacity of health, education, social protection and other workforces, to better support families, schools and communities, and to improve data, research and evidence (6).

The impact of the COVID-19 pandemic on children's mental health has raised serious concerns and has generated an enormous amount of speculation, media coverage and academic studies. So far, no event has aroused so much interest in the global media about the state of mental health as now. Part of the media coverage of COVID-19 has focused on disseminating information and raising awareness about the effects of the

\section{REFERENCES}

1. Kola L, Kohrt BA, Hanlon C, Naslund JA, Sikander S, Balaji M, et al. COVID-19 mental health impact and responses in low-income and middleincome countries: reimagining global mental health. Lancet Psychiatry. (2021) 8:535-50. doi: 10.1016/S2215-0366(21)00025-0

2. Fischer H-T, Elliot L, Bertrand SL. Guidance Note: Protection of Children During Infectious. The Alliance for Child Protection in Humanitarian Action. (2018). Available online at: https://resourcecentre.savethechildren.net/ document/guidance-note-protection-children-during-infectious-outbreaks/ (accessed November 10, 2021).

3. United Nations (UN). Policy Brief: The Impact of COVID-19 on Children. (2020). Available online at: https://unsdg.un.org/resources/policy-briefimpact-covid-19-children (accessed December 17, 2021).

4. Vieta E, Pérez V, Arango C. Psychiatry in the aftermath of COVID-19. Rev Psiquiatr Salud Ment. (2020) 13:105-10. doi: 10.1016/j.rpsm.2020.04.004

5. Brooks SK, Webster RK, Smith LE, Woodland L, Wessely S, Greenberg N, et al. The psychological impact of quarantine and how to reduce it: rapid review of the evidence. Lancet. (2020) 395:912-20. doi: 10.1016/S0140-6736(20)30460-8

6. United Nations Children's Fund (UNICEF). The State of the World's Children 2021: On My Mind_Promoting, Protecting and Caring for Children's Mental pandemic on mental health, however, a framework for action is needed (39).

This opinion article aimed to deepen knowledge and raise awareness of the key cultural and contextual factors affecting children and advocate for responses that improve children's lives in the current context. Hence, after governments, academics, and other stakeholders have shown a public commitment and broad communication to better mental health, we crucially conclude by calling governments, health institutions, and non-governmental organizations to commit to act in key areas and prioritize the needs of children and young people in any implementation of COVID-19 responses. If children's needs are not appropriately addressed, the mental health consequences for a generation of children could far exceed the immediate health and economic impact of the COVID-19 pandemic, leaving long-term social and economic consequences (22).

\section{AUTHOR'S NOTE}

The content of this manuscript has been presented in part at the UNICEF report on The State of the World's Children 2021 (6).

\section{AUTHOR CONTRIBUTIONS}

The author confirms being the sole contributor of this work and has approved it for publication.

\section{FUNDING}

MS receives grant support from the Alicia Koplowitz Foundation.

\section{ACKNOWLEDGMENTS}

The author would like to thank Inmaculada Baeza, MD PhD, Gisela Sugranyes, MD PhD, and Luisa Lazaro, MD PhD, of Hospital Clínic de Barcelona, for their guidance and mentoring.

Health, New York. (2021). Available online at: https://www.unicef.org/reports/ state-worlds-children-2021 (accessed January 6, 2022).

7. Gromada A, Rees G, Chzhen Y, UNICEF. Worlds of Influence: Understanding What Shapes Child Well-Being in Rich Countries. New York, NY: UNICEF 68.

8. Patel V, Saxena S, Lund C, Thornicroft G, Baingana F, Bolton P, et al. The Lancet Commission on global mental health and sustainable development. Lancet. (2018) 392:1553-98. doi: 10.1016/S0140-6736(18)31 $612-\mathrm{X}$

9. UNICEF Office of Research-Innocenti. Beyond Masks: Societal Impacts of COVID-19 and Accelerated Solutions for Children and Adolescents. Innocenti Research Report, Florence (2020). Available online at: https://www.unicefirc.org/publications/1149- beyond-masks-societal-impacts-of-covid-19and-accelerated-solutions-for-children-and-adolescents.html (accessed November 10, 2021).

10. Bronfenbrenner U. The Ecology of Human Development: Experiments by Nature and Design. Cambridge, MA: Harvard University Press (1979).

11. World Health Organization and Calouste Gulbenkian Foundation. Social Determinants of Mental Health. Geneva: World Health Organization (2014).

12. Nelson CA, Bhutta ZA, Burke Harris N, Danese A, Samara M. Adversity in childhood is linked to mental and physical health throughout life. BMJ. (2020) 371:m3048. doi: 10.1136/bmj.m3048 
13. Scott S, Briskman J, O'Connor TG. Early prevention of antisocial personality: long-term follow-up of two randomized controlled trials comparing indicated and selective approaches. Am J Psychiatry. (2014) 171:649-57. doi: 10.1176/appi.ajp.2014.13050697

14. Whittle S, Simmons JG, Dennison M, Vijayakumar N, Schwartz O, Yap $\mathrm{MBH}$, et al. Positive parenting predicts the development of adolescent brain structure: a longitudinal study. Dev Cogn Neurosci. (2014) 8:7-17. doi: 10.1016/j.dcn.2013.10.006

15. Viner RM, Ozer EM, Denny S, Marmot M, Resnick M, Fatusi A, et al. Adolescence and the social determinants of health. Lancet. (2012) 379:164152. doi: 10.1016/S0140-6736(12)60149-4

16. Brooks-Gunn J, Duncan GJ. The effects of poverty on children. Fut Children. (1997) 7:55-71. doi: 10.2307/1602387

17. Yadav UN, Rayamajhee B, Mistry SK, Parsekar SS, Mishra SK. A syndemic perspective on the management of non-communicable diseases amid the COVID-19 pandemic in low- and middle-income countries. Front Public Health. (2020) 8:508. doi: 10.3389/fpubh.2020.00508

18. Fore HH. A wake-up call: COVID-19 and its impact on children's health and wellbeing. Lancet Global Health. (2020) 8:e861-2. doi: 10.1016/S2214-109X(20)30238-2

19. Kostyrka-Allchorne K, Creswell C, Byford S, Day C, Goldsmith K, Koch M, et al. Supporting parents and kids through lockdown experiences (SPARKLE): A digital parenting support app implemented in an ongoing general population cohort study during the COVID-19 pandemic: a structured summary of a study protocol for a randomised controlled. Trials. (2021) 22:267. doi: 10.1186/s13063-021-05226-4

20. Barry MM, Clarke AM, Dowling K. Promoting social and emotional well-being in schools. Health Educ. (2017) 117:434-51. doi: 10.1108/HE-11-2016-0057

21. Williams I, Vaisey A, Patton G, Sanci L. The effectiveness, feasibility and scalability of the school platform in adolescent mental healthcare. Curr Opin. Psychiatry. (2020) 33:391-6. doi: 10.1097/YCO.0000000000000619

22. Solerdelcoll M, Arango C, Sugranyes G. Calling for the integration of children's mental health and protection into COVID-19 responses. Rev Psiquiatr Salud Mental. (2021) 14:113-6. doi: 10.1016/j.rpsm.2021.02.004

23. Corrigan PW, Morris SB, Michaels PJ, Rafacz JD, Rüsch N. Challenging the public stigma of mental illness: a meta-analysis of outcome studies. Psychiatric Serv. (2012) 63:963-73. doi: 10.1176/appi.ps.201100529

24. Thornicroft G, Rose D, Kassam A, Sartorius N. Stigma: ignorance, prejudice or discrimination? Br J Psychiatry. (2007) 190:192-3. doi: 10.1192/bjp.bp.106.025791

25. Hatzenbuehler ML. Structural stigma: Research evidence and implications for psychological science. Am Psychol. (2016) 71:742-51. doi: $10.1037 / \mathrm{amp} 0000068$

26. Sue DW, Capodilupo CM, Torino GC, Bucceri JM, Holder A, Nadal KL, et al. Racial microaggressions in everyday life: implications for clinical practice. Am Psychol. (2007) 62:271. doi: 10.1037/0003-066X.62.4.271

27. Trent M, Dooley DG, Dougé J, American Academy of Pediatrics Section on Adolescent Health, American Academy of Pediatrics Council on Community Pediatrics, American Academy of Pediatrics Committee on Adolescence, et al. The impact of racism on child and adolescent health. Pediatrics. (2019) 144(2). doi: 10.1542/peds.2019-1765

28. Cénat JM. How to provide anti-racist mental health care. Lancet Psychiatry. (2020) 7:929-31. doi: 10.1016/S2215-0366(20)30309-6

29. Ungar M, Theron L. Resilience and mental health: how multisystemic processes contribute to positive outcomes. Lancet Psychiatry. (2020) 7:441-8. doi: 10.1016/S2215-0366(19)30434-1
30. Ungar M. Resilience across cultures. Br J Social Work. (2008) 38:218-35 doi: 10.1093/bjsw/bcl343

31. Ungar M, Brown M, Liebenberg L, Othman R, Kwong WMŠ, Armstrong MI, et al. Unique pathways to resilience across cultures. Adolescence. (2007) 42:287-310.

32. Choudhry FR, Khan TM, Park MS-A, Golden KJ. Mental health conceptualization and resilience factors in the kalasha youth: an indigenous ethnic and religious minority community in Pakistan. Front Public Health. (2018) 6:187. doi: 10.3389/fpubh.2018.00187

33. Moreno C, Wykes T, Galderisi S, Nordentoft M, Crossley N, Jones $\mathrm{N}$, et al. How mental health care should change as a consequence of the COVID-19 pandemic. Lancet Psychiatry. (2020) 7:813-24. doi: 10.1016/S2215-0366(20)30307-2

34. Waite P, Pearcey S, Shum A, Raw JAL, Patalay P, Creswell C. How did the mental health symptoms of children and adolescents change over early lockdown during the COVID-19 pandemic in the UK? JCPP Adv. (2021) 1:e12009. doi: $10.1111 /$ jcv2.12009

35. Loades ME, Chatburn E, Higson-Sweeney N, Reynolds S, Shafran R, Brigden A, et al. Rapid systematic review: the impact of social isolation and loneliness on the mental health of children and adolescents in the context of COVID-19. J Am Acad Child Adolesc Psychiatry. (2020) 59:1218-39. doi: 10.1016/j.jaac.2020.05.009

36. Bunn S, Lewis S. Children's Mental Health and the COVID-19 Pandemic The Parliamentary Office of Science and Technology. (2021). POST PN 65. Available online at: https://post.parliament.uk/research-briefings/postpn-0653/ (accessed November 10, 2021).

37. Fritz J, de Graaff AM, Caisley H, van Harmelen A-L, Wilkinson PO. A systematic review of amenable resilience factors that moderate and/or mediate the relationship between childhood adversity and mental health in young people. Front Psychiatry. (2018) 9:230. doi: 10.3389/fpsyt.2018. 00230

38. Ungar M. Practitioner review: diagnosing childhood resilience-a systemic approach to the diagnosis of adaptation in adverse social and physical ecologies. J Child Psychol Psychiatry. (2015) 56:4-17. doi: 10.1111/jcpp. 12306

39. Su Z, McDonnell D, Wen J, Kozak M, Abbas J, Šegalo S, et al Mental health consequences of COVID-19 media coverage: the need for effective crisis communication practices. Global Health. (2021) 17:4. doi: 10.1186/s12992-020-00654-4

Conflict of Interest: The author declares that the research was conducted in the absence of any commercial or financial relationships that could be construed as a potential conflict of interest.

Publisher's Note: All claims expressed in this article are solely those of the authors and do not necessarily represent those of their affiliated organizations, or those of the publisher, the editors and the reviewers. Any product that may be evaluated in this article, or claim that may be made by its manufacturer, is not guaranteed or endorsed by the publisher.

Copyright (C) 2022 Solerdelcoll. This is an open-access article distributed under the terms of the Creative Commons Attribution License (CC BY). The use, distribution or reproduction in other forums is permitted, provided the original author(s) and the copyright owner(s) are credited and that the original publication in this journal is cited, in accordance with accepted academic practice. No use, distribution or reproduction is permitted which does not comply with these terms. 